\title{
Guiding Principles for a Pediatric Neurology ICU (neuroPICU) Bedside Multimodal Monitor
}

\section{Findings from an International Working Group}

Zachary M Grinspan 1,2,3; Yonina C. Eldar4; Daniel Gopher5; Amihai Gottlieb5; Rotem Lammfromm; Halinder S Mangat ${ }^{3,6}$; Nimrod

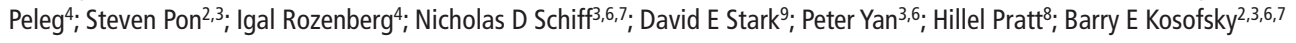

${ }^{1}$ Department of Healthcare Policy \& Research, Weill Cornell Medicine, New York, NY;

${ }^{2}$ Department of Pediatrics, Weill Cornell Medicine, New York, NY;

${ }^{3}$ New York-Presbyterian Hospital, New York, NY;

${ }^{4}$ Faculty of Electrical Engineering, Technion Israel Institute of Technology, Haifa, Israel;

${ }^{5}$ Faculty of Industrial Engineering and Management, Technion Israel Institute of Technology, Haifa, Israel;

${ }^{6}$ Department of Neurology, Weill Cornell Medicine, New York, NY;

${ }^{7}$ Brain and Mind Research Institute, Weill Cornell Medical College, New York, NY;

${ }^{8}$ Faculties of Medicine and Biomedical Engineering, Technion Israel Institute of Technology, Haifa, Israel;

${ }^{9}$ Departments of Medicine and Bioengineering, Stanford University, Stanford, CA

\section{Keywords}

Pediatric intensive care units [N02.278.388.493.390], neurology [H02.403.600], biomedical engineering [H02.070], informatics [L01.313], data display [F02.784.412.221]

\section{Summary}

Background: Physicians caring for children with serious acute neurologic disease must process overwhelming amounts of physiological and medical information. Strategies to optimize real time display of this information are understudied.

Objectives: Our goal was to engage clinical and engineering experts to develop guiding principles for creating a pediatric neurology intensive care unit (neuroPICU) monitor that integrates and displays data from multiple sources in an intuitive and informative manner.

Methods: To accomplish this goal, an international group of physicians and engineers communicated regularly for one year. We integrated findings from clinical observations, interviews, a survey, signal processing, and visualization exercises to develop a concept for a neuroPICU display.

Results: Key conclusions from our efforts include: (1) A neuroPICU display should support (a) rapid review of retrospective time series (i.e. cardiac, pulmonary, and neurologic physiology data), (b) rapidly modifiable formats for viewing that data according to the specialty of the reviewer, and (c) communication of the degree of risk of clinical decline. (2) Specialized visualizations of physiologic parameters can highlight abnormalities in multivariable temporal data. Examples include 3-D stacked spider plots and color coded time series plots. (3) Visual summaries of EEG with spectral tools (i.e. hemispheric asymmetry and median power) can highlight seizures via patient-specific "fingerprints." (4) Intuitive displays should emphasize subsets of physiology and processed EEG data to provide a rapid gestalt of the current status and medical stability of a patient.

Conclusions: A well-designed neuroPICU display must present multiple datasets in dynamic, flexible, and informative views to accommodate clinicians from multiple disciplines in a variety of clinical scenarios. 


\section{Correspondence to:}

Zachary M Grinspan, MD MS

Weill Cornell Medicine

Department of Healthcare Policy \& Research

402 East 67th Street

New York, NY 10065

Email: zag9005@med.cornell.edu
Appl Clin Inform 2016; 7: 380-398

http://dx.doi.org/10.4338/ACI-2015-12-RA-0177

received: December 21, 2015

accepted: February 29, 2016

published: May 18, 2016

Citation: Grinspan ZM, Eldar YC, Gopher D, Gottlieb A, Lammfromm R, Mangat HS, Peleg N, Pon S, Rozenberg I, Schiff ND, Stark DE, Yan P, Pratt H, Kosofsky BE. Guiding principles for a pediatric neurology ICU (neuroPICU) bedside multimodal monitor: Findings from an international working group. Appl Clin Inform 2016; 7: 380-398

http://dx.doi.org/10.4338/ACI-2015-12-RA-0177 


\section{Background and Objectives}

Pediatric neurocritical care is an emerging discipline that focuses on the care of children with serious acute neurologic diseases, such as traumatic brain injury, unremitting seizures (status epilepticus), and intracranial hemorrhage [1-3]. Caring for these patients demands that physicians integrate multiple sources of complex data in real time at the bedside [4]. For example, many children treated in a pediatric neurology intensive care unit (neuroPICU) require both routine physiologic monitoring for cardio-respiratory support as well as specific brain monitoring of intracranial pressure (ICP), cerebral perfusion pressure (CPP), brain tissue oxygenation, and neuronal activity (electroencephalography; EEG). Unlike typical medical ICU patients, for whom therapy focuses on maintaining systemic physiologic parameters (i.e. blood pressure, heart rate, blood oxygenation, etc.), therapy in neurologic ICU patients additionally requires coordinated regulation of these neurophysiologic parameters to optimize clinical outcomes $[4,5]$. The ICU physician must interpret these systemic and neurologic physiologic parameters on a moment to moment basis in the context of the patient's current medications, laboratory and imaging data, and overall clinical status. Adding further complexity, a pediatric ICU physician must also consider physiologic differences across the developmental spectrum - from newborns to adolescents. "Normal" values of physiologic parameters vary by age. Furthermore, patients in different age groups may require radically different treatments and therapeutic goals, even for the same disease process.

Several hurdles prevent full use of available clinical data at the point of care. For example, (1) the volume of data itself can be overwhelming [6]; (2) some of the data, EEG in particular, requires complex interpretation by physicians who are usually not at the bedside; (3) the data are collected via multiple proprietary devices; and (4) pediatric neurocritical care is a recently developed field, with only a small cadre of bona fide experts and few evidence-based recommendations to define the standard of care [1-3].

These hurdles - particularly related to integration of the EEG findings into real time clinical data analyses and decision-making - predispose pediatric neurology ICU patients to suboptimal care as well as medical errors. Our clinical experience includes several examples of these errors. In children with seizures who required anti-epileptic medication, we recall cases of under-treatment (i.e., a 10 hour delay in detection of non-convulsive seizures) and over-treatment (i.e., failure to reduce the rate of pentobarbital infusion after complete suppression of the EEG). In patients with brain injury, we recall cases of delayed treatment of poor blood flow to part of the brain (i.e., unappreciated EEG changes preceding vasospasm following subarachnoid hemorrhage) as well as cases of delayed treatment of poor blood flow to the whole brain (i.e., poorly controlled spikes in intracranial pressure following traumatic brain injury). A recent systematic review confirmed our clinical experience - it described the high prevalence of medical errors in ICU settings and their association with worse clinical outcomes [7].

Automated computer analyses of the relevant neurophysiologic parameters are unlikely to replace human interpretation in the near future, particularly for EEG data. Current EEG processing algorithms remain inferior to human interpretation of the raw EEG tracings. In clinical practice, patient care requires ongoing input from physicians skilled in identifying underlying patterns of brain activity [8-10]. Furthermore, seizure detection is not a "solvable" signal analysis problem; some patterns of EEG activity may represent either an ictal (i.e., seizure) or an inter-ictal (i.e., not seizure) state [11, 12 ], and can only be interpreted in the clinical context, or based on response to treatment with antiepileptic medications.

This paper describes our interdisciplinary project in which experts from several clinical and engineering disciplines joined together to form a working group. Our goal was to outline guiding principles for the creation of a neuroPICU monitor that integrates and displays data from multiple sources in an intuitive and informative manner. 


\section{Methods}

\subsection{Setting}

From August 2012 through September 2013, experts from Weill Cornell Medical College in New York, NY and Technion - Israel Institute of Technology in Haifa, Israel communicated regularly via teleconference to discuss design principles for a pediatric neurology intensive care unit (neuroPI$\mathrm{CU})$ bedside monitor that would integrate data from multiple sources. The Weill Cornell IRB reviewed and approved all studies. All clinical data reviewed by investigators at Technion was fully deidentified.

\subsection{Focus and scope}

After our initial teleconferences, we selected three areas of focus. First, we explored the desired characteristics of a neuroPICU dashboard using bedside observations, interviews of physicians, and a survey of physicians. Second, we created and compared visualization tools to explore creative ways to view and manipulate complex medical data. Third, we applied principles from the human factors engineering literature to conceptualize how information derived from multiple sources might be arranged on an integrated bedside display.

\subsection{Needs assessment - Observations \& Interviews}

A graduate student (RL) from the Technion conducted bedside observations and interviews with several practicing physicians in order to understand what "information gaps" practitioners identified in their needs for integrating and displaying bedside monitoring data. She conducted 25 hours of in-person observations of clinical care at the NewYork-Presbyterian Hospital/Weill Cornell Medical Center in four settings (adult neurologic ICU, pediatric ICU, general pediatric neurology, and pediatric epilepsy). She also conducted eight hours of interviews with eight experts (two medical informaticists, and six physicians with expertise in multiple specialties: neurology critical care, pediatric critical care, general pediatrics, clinical neurophysiology, pediatric neurology). She recorded her experiences using hand written field notes. She used a de-identified style (initials instead of patient names; "Day 1", "Day 2" instead of actual dates) in order to ensure her notes were compliant with legal regulations for de-identified data [13].

She then summarized her findings, and extracted 2-3 key themes from her experience, using a thematic analysis approach [14]. To increase the credibility of her observations, these themes were reviewed, discussed, and refined with experts in relevant clinical subdomains (DS, BK general pediatric neurology; ZG pediatric epilepsy; SP pediatric ICU; HM adult neurologic ICU) and experts in human factors engineering (DG). We present a brief narrative summary of these themes.

\subsection{Needs assessment - Survey}

We developed a survey to assess physicians' priorities for data required to optimally manage patients in a neuroPICU in three mock clinical scenarios: status epilepticus, increased intracranial pressure (ICP), and subarachnoid hemorrhage (Appendix 1). In the survey, we presented a brief clinical history, then asked each physician to choose the ten data elements deemed most appropriate for clinical use for the management of each of those mock patients, from a list of 43 possible data elements in seven categories (vital signs, ICP measurements, neurophysiologic and EEG parameters, medications, ventilator settings, and laboratory values; Appendix 2). These 43 data elements were selected by brainstorming and consensus among five physicians in the group (ZG, BK, SP, DS, HM). Subjects were provided a text box to add additional data elements. We administered the survey to a convenience sample of pediatricians, pediatric neurologists, pediatric ICU specialists, and pediatric neurosurgeons at two academic medical centers in New York City.

We performed two comparisons. First, we compared the top ten preferred data elements in the ICP scenario between pediatric neurologists and pediatric ICU physicians. To do so, we counted how often physicians in each group indicated a preference for a particular data element. We then 
listed the top ten, in order, for each group. We interpreted that a group of physicians felt the data element was relevant if it appeared on the list of top ten. We then interpreted the rank of the data element to imply the relative importance of the data element.

Second, we compared the top five preferred data elements in each of the three clinical scenarios, among the entire sample of surveyed physicians. To do so, we counted how often physicians indicated a preference for a particular data element, from the entire sample of physicians. We then listed the top five, in order, for each scenario.

\subsection{Visualization - physiology}

We created a 3-dimensional visualization of physiology data using a de-identified dataset with time series data $(85 \mathrm{~Hz})$ on five parameters: ICP, mean arterial pressure (MAP), cerebral perfusion pressure (CPP), heart rate (HR), and end tidal CO2 capnography $(\mathrm{CPN})$. The data were collected from an adult man who developed increased intracranial pressure due to traumatic brain injury. A neurosurgically placed intracranial monitor (Codman ICP Express, DePuy Synthes, Raynham, MA) collected ICP data. MAP and HR were measured continuously via an arterial line. CPN was measured on exhaled air using a capnography device on the ventilator. CPP is a derived value: CPP = MAP - ICP. The graphics were created using the "Gulrit" CAD software package [15].

\subsection{Visualization -- EEG Signal Processing}

We developed a method to use spectral analysis of EEGs to visually identify seizures. We obtained several clinical EEG recordings from children who had electrographic seizures from our local teaching files. The EEG recordings were captured with the XLTEK system (Natus Medical Inc, San Carlos, CA) using a standard 10-20 montage with 19 channels of EEG $(256 \mathrm{~Hz})$. These files were exported into CSV format, and manually de-identified by removing all patient information, and shifting each date-time stamp by an arbitrary fixed amount. Signal processing was performed using Matlab software (Mathworks Inc, Natick, MA). In this report, we demonstrate two examples of the signal processing techniques we employed. We selected these techniques based on discussion and consensus, drawing on two sources of intuition: our clinical experience visually interpreting the EEG (ZG) and our research experience in signal processing (YE, NP, IR, NS, HP). Of importance, these are not validated techniques, and would need rigorous evaluation prior to use in patient care.

\section{Preprocessing}

Each raw EEG signal was passed through two filters: (1) a bandpass filter (1Hz to $71 \mathrm{~Hz})$ in order to minimize low and high frequency noise, and (2) a notch filter $(60 \mathrm{~Hz}$, bandwidth $1.3 \mathrm{~Hz})$ to remove power grid contamination of the signals. We then performed a Short Time Fourier Transform (STFT; 2 second Hamming windows with 1 second overlap) on each channel of EEG. Thus each of the 19 EEG signals was transformed into a series of discrete power spectra at 1-second intervals.

\section{Hemispheric Asymmetry}

We reproduced a variant of the previously described "hemispheric asymmetry" spectrogram [16] on a record containing a left temporal lobe seizure. To do so, we calculated the log of the absolute value of the arithmetic difference of the power spectra between the left and right temporal lobes. We conceptualized this visualization as a "focal seizure detector", because focal seizures typically produce large increases in power, spatially restricted to a subset of EEG leads.

\section{Median Power}

We wanted to visualize the "average" power spectrogram across the head. However, instead of the mean power, we displayed the median power in order to control for electrode artifact. For example, if a single electrode falls off the head during recording, the mean power will vary substantially, whereas the median power will remain largely unaffected. We created the spectrogram as follows: for each 1-second time period, we calculated the median power across all 19 channels in each frequency bin. We conceptualized this visualization as a "generalized seizure detector", because generalized 
seizures typically consist of an abrupt increase in EEG power, which can detected throughout the full set of EEG leads.

\subsection{Conceptualization of the Display}

Integrating the results from the survey, observations, interviews, 3-D visualizations, and EEG signal processing exercises, we conceptualized a layout of a bedside display. In addition to our own observations, we also relied on published reviews addressing similar topics, including a summary of pediatric-specific demands of an electronic health record [17] and a recent review of clinical neuroinformatics [18].

\section{Results}

\subsection{Needs Assessment - Observations \& Interviews}

Two key themes emerged from thematic analysis of the observations and interviews, summarized as follows.

First, there are different kinds of information gaps that a bedside monitor can fill, to answer three types of questions:

(a) Retrospective Review (What happened overnight?) Allow a bedside physician to rapidly sift through 12-24 hours of time series data, particularly to understand overnight events.

(b) Current Status (What is going on now?) Provide live reporting of relevant, patient-specific data.

(c) Degree of Risk of Clinical Decline (Is there an impending catastrophe?) Alert physicians to early signs of imminent clinical decline.

Second, clinicians use two complimentary strategies to think about critically ill patients: "thinking in problems" and "thinking in systems". Senior clinicians often frame a patient's issues in terms of specific problems, whereas nurses and junior clinicians (ICU hospitalists, physician assistants, interns, residents, fellows) often think about a patient's individual organ systems. Each of these different modes is clinically important. The senior clinician designs the overall strategy for diagnostic testing and treatment, whereas nurses and junior clinicians must methodically attend to all the details of complex care. For example, for an unresponsive, hypotensive patient with traumatic brain injury, the nurse or junior clinician may need to first think through the cardiovascular, respiratory, and neurologic needs of the patient (i.e. systems), whereas the senior clinician may focus directly on hypotension, apnea, and increased intracranial pressure (i.e. problems). In practice, clinicians often employ both strategies at different times, even for the same patient.

\subsection{Needs Assessment - Survey}

38 physicians responded to the survey, including 17 pediatric critical care physicians, 15 pediatric neurologists, two pediatric neurosurgeons, and four general pediatricians. A subset of 27 physicians responded to items about the subarachnoid hemorrhage scenario.

\section{Pediatric Critical Care vs Pediatric Neurology}

In the intracranial pressure scenario, there were qualitatively three kinds of agreement between pediatric critical care and pediatric neurology physicians on the importance of data elements.

1. Agreement on importance and relevance. For example, both groups agreed that measuring intracranial pressure was the most important parameter.

2. Agreement on relevance but disagreement on importance. For example, both groups agreed that the serum sodium was relevant, but disagreed on its importance.

3. Disagreement on relevance. For example, the pediatric critical care physicians indicated that a blood gas was relevant, but the pediatric neurologists did not. ( $>$ Table 1 ) 


\section{Different Scenarios}

There was little overlap between the top five requested data elements in the three scenarios. No data element appeared in the top five for all three scenarios. ( $\triangleright$ Table 2$)$

\subsection{Visualization - Physiology}

We created two visualizations to illustrate possibilities for the integrated display of temporal information derived from multiple data streams. First, we created a "stacked spider plot", in which we plotted five physiologic parameters as a 3-dimensional manipulable object. Each time series was normalized, and averaged into equal sized time periods. For each time point, the values were plotted as a five-sided spider plot $(\triangleright$ Figure 1A). These spider plots were then stacked, to create a 3-dimentional object, which could be manipulated by the user. Qualitatively, this display made a transient increase in ICP (Lundberg type A wave) visually apparent ( $\triangleright$ Figure 1B), though the relationships between variables were not always clear. Connecting the vertices for selected variables highlighted changes over time ( $\triangleright$ Figure $1 \mathrm{C}$ ).

Second, we created a "stacked color coded time series." ( Figure 2) Each value is plotted as a small black dot, with time on the $\mathrm{x}$-axis and the physiologic value on stacked $\mathrm{y}$-axes. Thresholds for critical values are labeled and highlighted by dotted lines, but no other values appear on the $y$-axes to avoid visual clutter. Each time series is plotted on a grey rectangular background, which visually separates one from the other. When values are abnormal, the background changes to red if too high, blue if too low. The degree of color change is roughly proportional to the degree of abnormality. The background is white when values are missing. In addition, the most recent values appear in a large font to the right of the time series, with abnormal values highlighted with color. Qualitatively, the Lundberg type A wave (transient increase in ICP) is again visually apparent. In this visualization, it is also clearer that during the A wave, the MAP rises in coordinate fashion, CPP occasionally dips below the critical threshold of 60 , heart rate rises and stays elevated, and the capnography reading is often unavailable.

\subsection{Visualization - EEG Signal Processing}

The median EEG power spectrogram produced a visually identifiable seizure "fingerprint" for an example of a generalized seizure ( $\triangleright$ Figure 3). Similarly, the hemispheric asymmetry spectrogram produced a visually identifiable seizure "fingerprint" for an example of a focal seizure ( $\triangleright$ Figure 4$)$. Harmonics of the dominant frequency appear as descending parallel bands in both spectrograms, strengthening the visual metaphor of a "fingerprint."

\subsection{Conceptualization}

We conceptualized the layout of a bedside monitor using the celestial metaphor "the sun, the moon, and the stars" ( $\triangleright$ Figure 5). The sun and moon, in this case, are large areas in the center of the screen, which represent the most important sets of variables: (a) customizable subsets of continuously measured physiologic and neurophysiologic parameters, and (b) processed EEG signals. The stars represent additional customizable variables, which appear on the periphery of the screen. Such variables might include laboratory values, other vitals signs, or medication information.

\section{Discussion}

\subsection{Summary \& Synthesis}

We performed a series of exercises, including clinical observations, interviews, a survey, signal processing, 3-D visualizations, and conceptualization of a display, in order to develop guiding principles for a pediatric neurology ICU bedside display, summarized here:

1. A bedside display must support three tasks:

a Rapid retrospective review of a patient's physiologic trends from the preceding 12-24 hours

@ Schattauer 2016 ZM Grinspan et al.: Guiding Principles for a Pediatric Neurology ICU Bedside Multimodal 
b Rapidly modifiable data views, both problem- and system-based, to assess and monitor the current status of a patient

c Communicate the degree of risk of clinical decline, including critical alarms

2. Effective visualizations should highlight abnormalities in time series data.

3. EEG can be visually summarized to highlight patient-specific seizure "fingerprints."

4. A display should emphasize subsets of physiologic and processed EEG data as is relevant to a particular patient.

\subsection{Three tasks}

\section{Rapid retrospective review}

In order to support retrospective review of multimodal data, multiple data sources need to be synchronized and stored, at high time resolution [18]. This has been challenging in practice, as many commercial ICU devices are not easily linked to share data, nor do they readily support time synchronization [18]. Once these technical and proprietary marketplace barriers are overcome, future work will need to assess the added value of synchronized, high time resolution ICU data.

\section{Rapidly modifiable data views}

Medical informatics pedagogy teaches that data may be viewed in source-oriented views (to gather data collected from the same place), time-oriented views (to see trends), and concept oriented views (by system or by problem) [19]. Our findings emphasize that each of these views may be relevant for different clinicians, at different times, even for the same patient. Although recent work suggests that there is a limited subset of data that is of highest value to a bedside clinician [20], our survey findings show that the specific subset of data varies by physician specialty, and by disease process.

\section{Degree of risk of clinical decline}

Although the importance of alarms in the ICU setting is self-evident, there are too many ICU alarms, not too few $[21,22]$. The proliferation of monitor and device alarms in the ICU leads to a large number of nuisance alarms, many of which do not require intervention. Such over-alerting leads to alarm fatigue, which may blind a physician to important changes in a patient's status [22]. A more nuanced role for a next-generation bedside monitor might include an alarm regulator that highlights relevant alarms and suppresses alarms likely to be false.

Of importance, the "degree of risk of clinical decline" of a patient is more than the sum total of relevant alarms, and serves a different purpose than an alarm tied to a single physiologic parameter. For example, automated extraction of data from the EHR and other information systems would allow real time calculation of validated risk scores, such as the Pediatric Early Warning Score (PEWS) [23], Pediatric Risk of Mortality Score (PRISM 3) [24], or Acute Physiology Score (APS) [25], each of which provides additional context for interpreting physiologic values. A single physiologic parameter alarm typically requires immediate action (i.e., low blood pressure prompts administration of a vasopressor medication); whereas these risk scores help clinicians understand the overall trajectory of each child, and prioritize which children require the most attention.

\subsection{Visualization - Physiology}

Spider plots (also called "radar plots"[26] or "polar-polygon plots"[27]) have been proposed to display neurologic ICU data [28], in order to allow the visual configuration of the data to improve recognition of patterns $[27,29]$. Our stacked spider plots extend this graphical technique to include the dimension of time, and illustrate how 3-D visualizations can improve understanding of multivariable relationships [30]. More traditional time series plots may also be effective, such as those included in our stacked color coded time series. Color coding and thoughtful selection of critical values can highlight abnormalities and help clarify relationships between variables. Further work is required to understand the relative merits of these and other techniques aimed at improving visualization of time series data. 


\subsection{Visualization - EEG}

Several techniques for visual summary of EEG have been described, including many in common use, such as compressed spectral array and amplitude integrated EEG [31]. Our proposed display focused on two spectral tools: one designed to identify focal seizures (hemispheric asymmetry spectrogram [16]), and one designed to identify generalized seizures (median power spectrogram). Each has potential advantages. Hemispheric asymmetry highlights periods of time with spatially restricted increases in power, typical for focal seizures. A median power spectrogram may detect generalized seizures, while simultaneously providing a visual display that is robust to artifacts caused by corrupted leads. Further research will be required to understand if the seizure fingerprints created by these techniques can effectively help bedside physicians detect seizures in their patients in real time.

\subsection{Display Concept}

With our "sun, moon, and stars" display concept, we mapped screen organization, location, centrality, and size of data elements to the relative importance and dynamic properties of each patient parameter [30, 32]. Furthermore, in order to facilitate the combined integration and detection of changes in dynamic physiological indices, we developed configural object displays [33, 34]. The integral display of the present work is only a first step, which should be more systematically studied and optimized.

\subsection{Limitations}

Each described exercise had limitations. Our observations and interviews were focused on physicians' needs and did not include potential needs of other team members in the ICU, such as nurses, respiratory therapists, and EEG technologists. The external validity of the survey may be limited, given the small sample size, the constrained list of data elements provided to the subjects, and the convenience sample study design. The "stacked spider plots" need additional work to effectively show relationships between variables. We performed the EEG analysis on a few example EEGs, and the visualization of physiologic data from a single patient rather than a representative sample of each. The display concept will need several additional iterations before development of a working prototype.

\subsection{Future Work}

Two major areas of future work should grow from the findings of this working group. First, a prototype of a neuroPICU dashboard based on these principles should be built and evaluated. Second, human factors experiments should be conducted to understand the advantages and disadvantages of specific visual display concepts, such as stacked spider plots and stacked color coded time series for multivariable time series, hemispheric asymmetry spectrogram for focal seizure identification, and median EEG power for generalized seizure identification. Both endeavors - prototyping and research - will require ongoing collaborative efforts from informatics experts, biomedical engineers, and physicians with expertise in multiple domains.

\section{Conclusions}

Optimal display of data for critically ill children with neurologic injury has the potential to greatly enhance care for this vulnerable population. A well-designed neuroPICU dashboard must present multiple datasets in dynamic, flexible views to accommodate clinicians from multiple disciplines to better integrate data streaming from the bedside in real time in a variety of clinical scenarios. Our findings lay the groundwork to develop such displays. 


\section{Clinical Relevance}

Pediatric neurocritical care requires physicians to integrate overwhelming amounts of data from multiple sources. This work outlines guiding principles toward the design of a clinical dashboard that integrates multiple data sources to help physicians manage these complex and vulnerable patients at the bedside.

\section{Funding}

We are grateful to the Joan and Irwin Jacobs Technion-Cornell Innovation Institute for providing seed funding for this project. This project was supported in part by the National Institute for Neurologic Disease and Stroke grant \#K12-NS0662 (ZG).

\section{Conflicts Of Interest}

The authors declare that they have no conflicts of interest in the research.

\section{Protection Of Human Subjects}

The Weill Cornell IRB reviewed and approved all studies. All clinical data reviewed by investigators at Technion was fully de-identified. Animal subjects were not included in the project.

\section{Acknowledgements}

We thank the following individuals for their participation, assistance, and feedback with several components of this work: Baria Hafeez MS, Michael Bell MD, Rainu Kaushal MD MPH, Erika Abramson MD MS, Gershon Elbar PhD, Maor Grinberg MD, Craig Gottesman PhD, Tom Campion $\mathrm{PhD}$, Sameer Malhotra MD MA, Ramin Zabih PhD, Eli Appleboim PhD, Oded Sayar, and Tomer Caspi. 


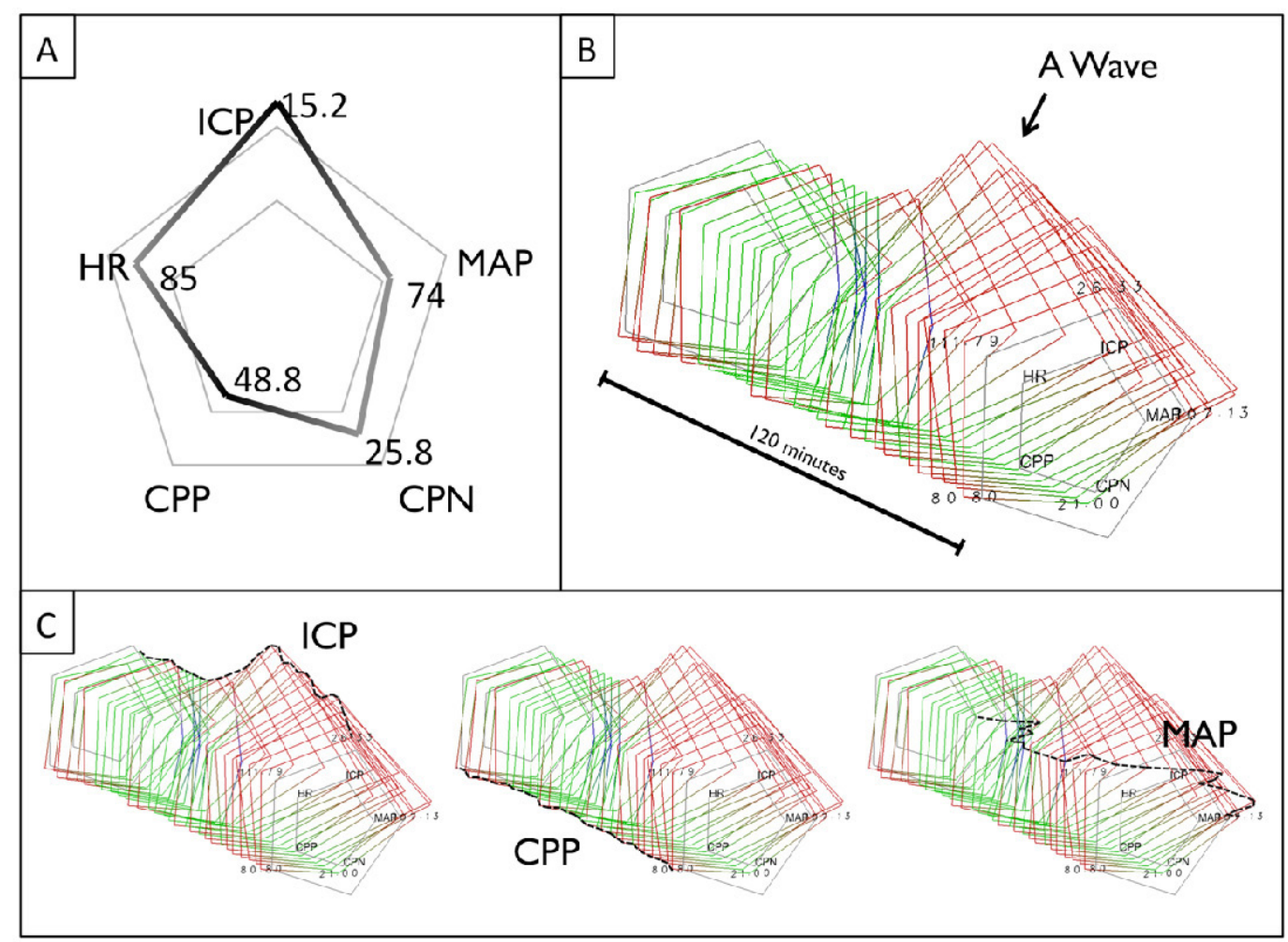

Fig. 1 Stacked Spider Plot. Panel A. Spider plot of five clinical variables averaged over five minutes, and normalized: ICP (intracranial pressure), MAP (mean arterial pressure), CPN (capnography), CPP (cerebral perfusion pressure), and HR (heart rate). Gray pentagons indicate lower and upper bounds of normal. Out of range variables are shaded a different color than normal variables. Panel B. 120 minutes of data represented in 24 stacked spider plots. A Lundberg type A wave (transient increase in ICP) is visually apparent. Panel $\mathrm{C}$. Connecting the vertices clarifies the time course of individual variables. The spike in ICP (dashed line, left) did not affect CPP (dashed line, center) because of commensurate changes in MAP (dashed line, right). 


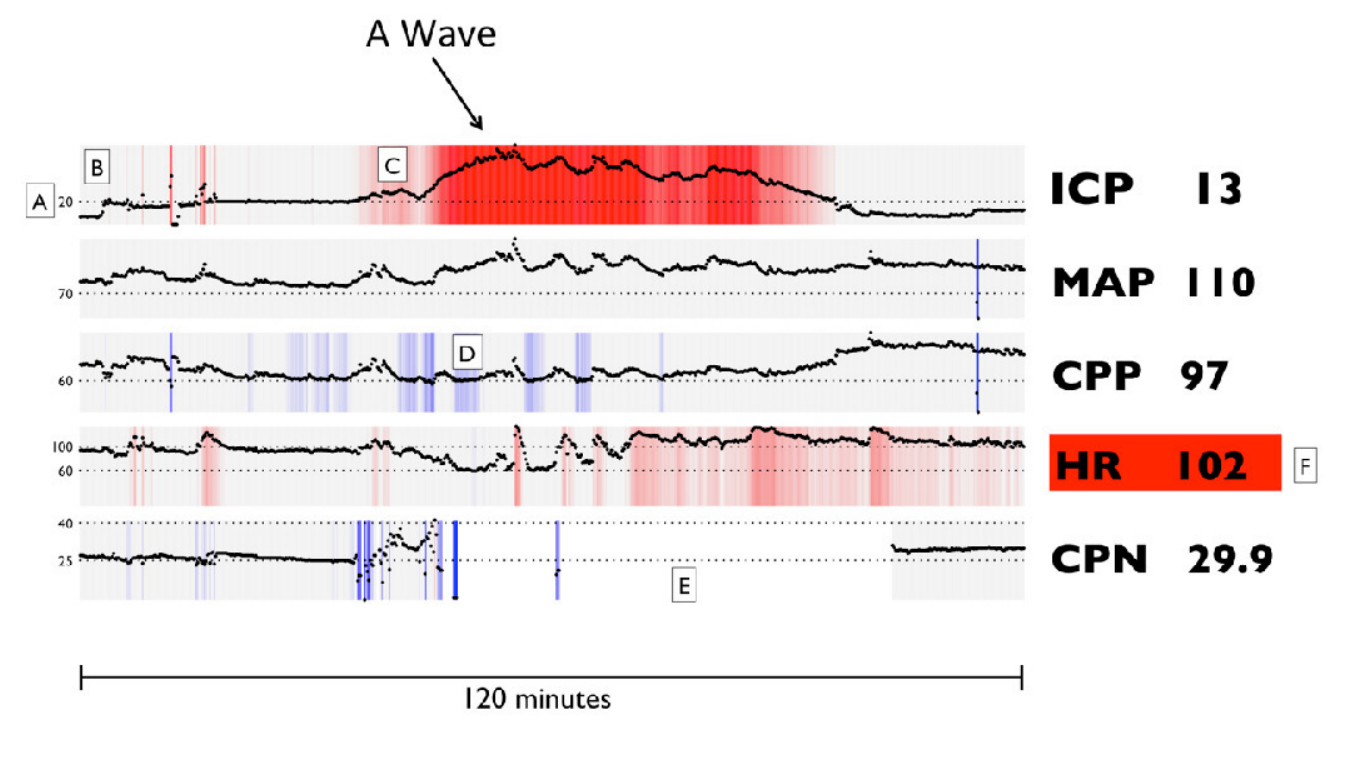

Fig. 2 Stacked color coded time series. Same data and abbreviations as in figure 1: ICP (intracranial pressure), MAP (mean arterial pressure), CPN (capnography), CPP (cerebral perfusion pressure), and HR (heart rate). Each value is plotted as a small black dot, time on the $x$-axis and the physiologic value on the $y$-axis. (A) Thresholds for critical values are labeled and highlighted by dotted lines. No other values appear on the $y$-axes. (B) Background for each time series is light grey, visually separating one from the other. (C, D) Abnormal values are highlighted by changing the background red when the value is too high, blue when too low. (E) Missing values are de-emphasized with a white background and no dots. (F) Current values appear in large sans-sarif font, with abnormal values highlighted. The Lundberg type A wave (transient increase in ICP) is again visually apparent. It is also clearer that during the A wave, the MAP rises in concert, CPP occasionally dips below the critical threshold of 60 , heart rate rises and stays elevated, and the capnography reading is unavailable. 

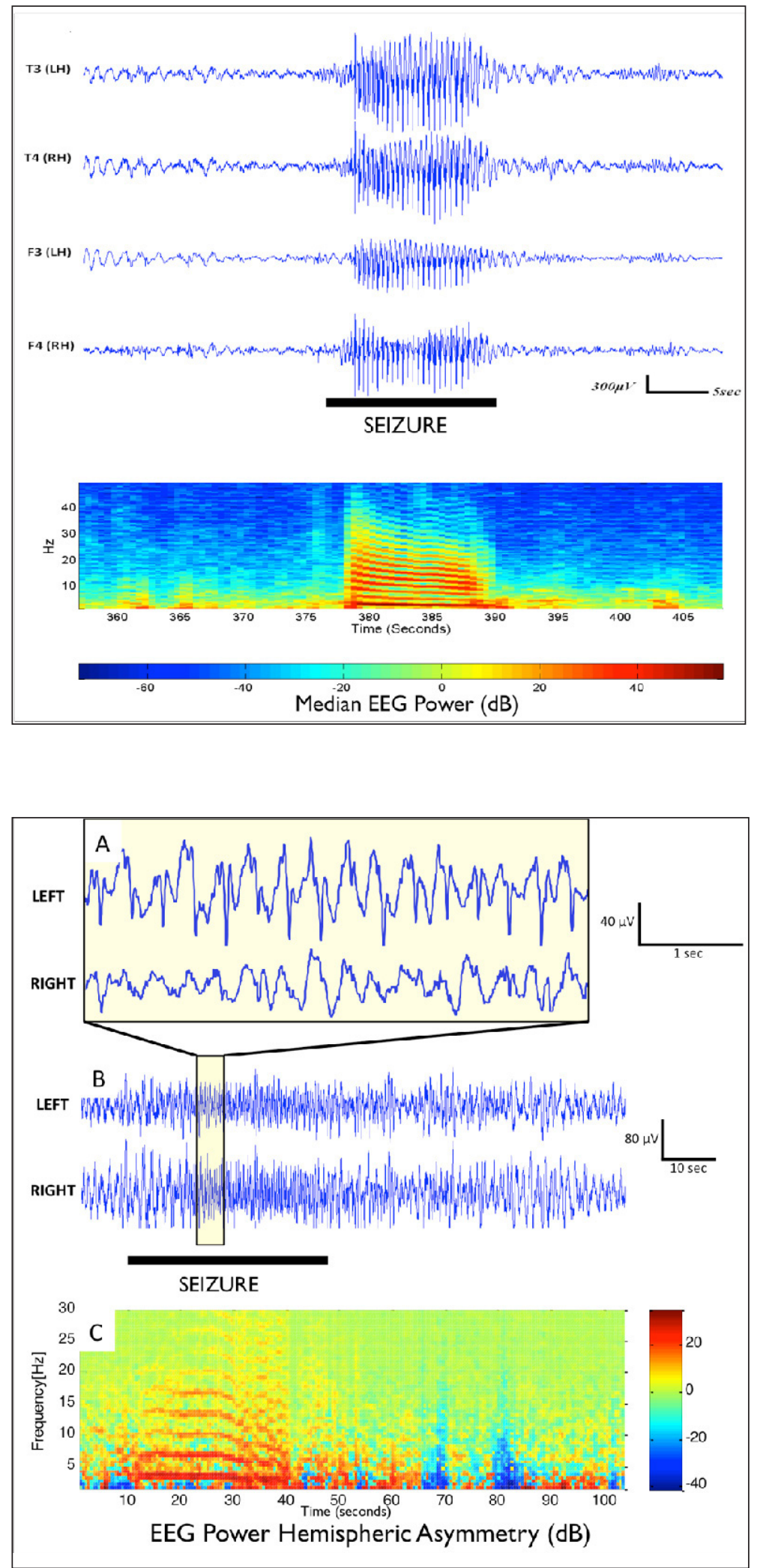

Fig. 3 Median EEG Power Spectrogram. Top. Four channels of surface EEG recording from the left $(T 3, F 3)$ and right $(T 4, F 4)$ hemispheres of a child with absence epilepsy. A seizure is indicated with the black horizontal line. Bottom. Median EEG power spectrogram demonstrates a clearly visible seizure fingerprint.

Fig. 4 EEG Power Hemispheric Asymmetry Spectrogram. A. EEG tracings from the left and right temporal lobes.

The left temporal traces show the regular spiking activity typical of a temporal lobe seizure. B. At a

compressed time frame, the seizure (horizontal bar) is not readily apparent. C. The hemispheric asymmetry spectrogram demonstrates a clearly visible seizure fingerprint. 


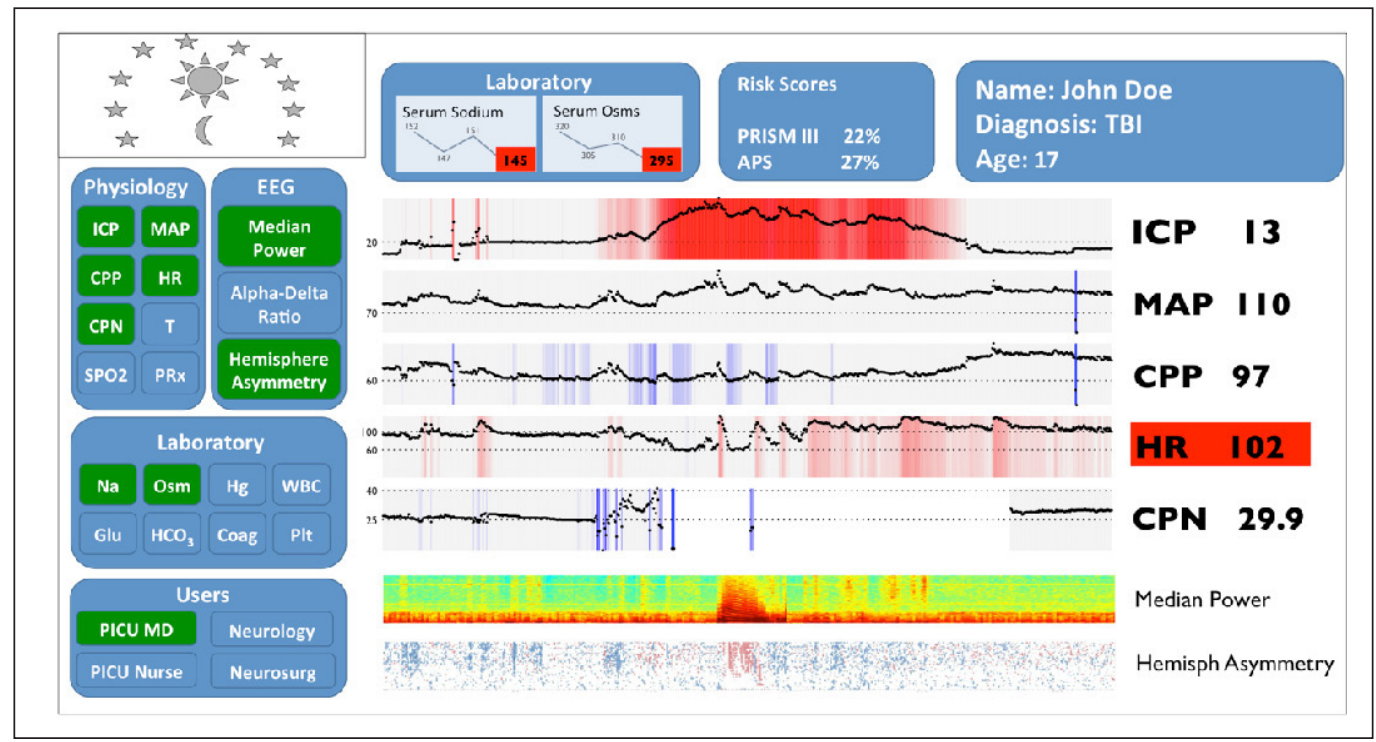

Fig. 5 Concept for a display. Inset. "Sun, Moon, and Stars" metaphor for the layout. Main. The physiology and EEG data are given visual prominence as the "sun" and "moon". The details of each can be customized by selecting variables. Additional relevant data can be found in the surrounding "stars", which may contain, for example, laboratory values or calculated risk scores. 
Table 1 Top 10 Data Elements Requested to Help Manage a Patient with Increased Intracranial Pressure, by Physician Type

\begin{tabular}{|l|l|}
\hline Pediatric Critical Care $(\mathbf{n = 1 7})$ & Pediatric Neurology $(\mathbf{n}=\mathbf{1 2})$ \\
\hline 1. Intracranial Pressure & 1. Intracranial Pressure \\
\hline 2. Sodium & 2. Respiratory Rate \\
\hline 3. Respiratory Rate & 3. Systolic BP \\
\hline 4. Cerebral Perfusion Pressure & 4. Cerebral Perfusion Pressure \\
\hline 5. Presence/Absence of Seizures & 5. Temperature \\
\hline 6. Systolic BP & 6. Sodium \\
\hline 7. Temperature & 7. Pulse Oximetry \\
\hline 8. Blood Gas & 8. Presence/Absence of Seizures \\
\hline 9. Mean BP & 9. Mean BP \\
\hline 10. List of Active Medications & 10. Infusion Rate of Sedative Meds \\
\hline
\end{tabular}

Table 2 Top 5 Data Elements Requested to Help Manage a Patient in Three Clinical Scenarios, Among All Surveyed Physicians.

\begin{tabular}{|l|l|l|}
\hline Increased Intracranial Pressure & Subarachnoid Hemorrhage & Status Epilepticus \\
\hline ( $\mathbf{n = 3 8}$ physicians) & ( $\mathbf{n = 2 7}$ physicians) & ( $\mathbf{n}=\mathbf{3 8}$ physicians) \\
\hline 1. Intracranial Pressure & 1. Systolic BP & 1. Presence/Absence of Seizures \\
\hline 2. Cerebral Perfusion Pressure & 2. Temperature & 2. Drug levels \\
\hline 3. Respiratory Rate & 3. Presence/Absence of Seizures & 3. List of Active Medications \\
\hline 4. Serum Sodium & 4. Pulse 0x & 4. Temperature \\
\hline 5. Systolic BP & 5. Heart Rate & 5. Infusion Rate of Sedative Meds \\
\hline
\end{tabular}




\section{Appendix 1 Three clinical scenarios and instructions used in the survey}

\section{Scenario 1. ICP}

You are at the bedside caring for an intubated child (7 years old) with increased intracranial pressure (ICP). A full team of physicians, residents, and nurses are also there in their respective roles. Check up to 10 data elements that will best help you in your typical clinical role to manage HIGH ICP.

\section{Scenario 2. Status Epilepticus}

You are at the bedside caring for an intubated child (7 years old) with status epilepticus. A full team of physicians, residents, and nurses are also there in their respective roles. Check up to 10 data elements that will best help you in your typical clinical role to manage STATUS EPILEPTICUS.

\section{Scenario 3. Stroke}

You are at the bedside caring for an intubated child (7 years old) with subarachnoid hemorrhage from a ruptured aneurysm. The aneurysm was successfully coiled. It is day 5 , and you are worried about vasospasm and stroke. The child is paralyzed, and so you cannot perform a clinical exam. A full team of physicians, residents, and nurses are also there in their respective roles. Check up to 10 data elements that will best help you in your typical clinical role to identify and treat STROKE. 


\section{Appendix 2}

\section{VITAL SIGNS / PHYSIOLOGY}

$\square$ Temperature

$\square \quad$ Heart Rate

$\square \quad$ Respiratory Rate

$\square$ Systolic BP

$\square \quad$ Diastolic BP

$\square \quad$ Mean BP

$\square \quad$ Pulse Ox

$\square \quad$ Water temp (Arctic Sun)

$\square$ Capnometry

$\square \quad$ Central Venous Pressure

$\square \quad$ Noninvasive Cardiac Output

\section{INTRACRANIAL PRESSURE}

Intracranial Pressure

$\square \quad$ Cerebral Perfusion Pressure

$\square \quad$ CSF Drainage

\section{NEUROPHYSIOLOGY}

Brain Tissue Oxygenation

$\square \quad$ Near-Infared Spectroscopy

$\square \quad$ Transcranial Doppler Results

EEG

Presence/absence of seizures

Sleep/wake cycling

$\square \quad$ Hemispheric Asymmetry

$\square \quad$ Raw traces

$\square \quad$ Alpha/delta ratio

\section{MEDICATIONS}

Infusion rate of sedative meds

$\square \quad$ Infusion rate of analgesic meds

$\square \quad$ Infusion rate of pressor meds

$\square \quad$ Infusion rate of hypertonic saline

$\square \quad$ List of active medications

$\square \quad$ Time of last hypertonic saline bolus

$\square$ Time of last mannitol bolus

\section{VENTILATOR}

Ventilator mode

$\square \quad$ Respiratory rate, machine

$\square$ Respiratory rate, patient

$\square$ Tidal Volume, inspiratory

$\square$ Tidal Volume, expiratory

$\square \quad \mathrm{FiO} 2$

$\square \quad$ Peak inspiratory pressure

$\square \quad$ Positive end expiratory pressure

$\square \quad$ Mean airway pressure

\section{LABORATORY}

Drug levels

$\square$ Sodium

$\square \quad$ Other electrolytes

$\square \quad$ Lactate

$\square \quad$ Blood gas

Are there other data elements you would like to see?

Appendix 2 Available choices for each scenario in the survey. Physicians were instructed to check up to 10 data elements in each clinical scenario. 


\section{References}

1. LaRovere KL, Riviello JJ, Jr. Emerging subspecialties in neurology: building a career and a field: pediatric neurocritical care. Neurology 2008; 70(22): e89-91. PubMed PMID: 18505973.

2. Scher M. Proposed cross-disciplinary training in pediatric neurointensive care. Pediatr Neurol 2008; 39(1): 1-5. PubMed PMID: 18555165. Epub 2008/06/17.

3. Murphy S. Pediatric neurocritical care. Neurotherapeutics 2012; 9(1): 3-16. PubMed PMID: 22183817. Pubmed Central PMCID: 3271153. Epub 2011/12/21.

4. Kochanek PM, Carney N, Adelson PD, Ashwal S, Bell MJ, Bratton S, Carson S, Chesnut RM, Ghajar J, Goldstein B, Grant GA, Kissoon N, Peterson K, Selden NR, Tasker RC, Tong KA, Vavilala MS, Wainwright MS, Warden CR. Guidelines for the acute medical management of severe traumatic brain injury in infants, children, and adolescents--second edition. Pediatr Crit Care Med 2012; 13 (Suppl. 1): S1-S82. PubMed PMID: 22217782. Epub 2012/01/11.

5. Stein SC, Georgoff P, Meghan S, Mirza KL, El Falaky OM. Relationship of aggressive monitoring and treatment to improved outcomes in severe traumatic brain injury. Journal of neurosurgery 201; 112(5): 1105-1112. PubMed PMID: 19747054. Epub 2009/09/15.

6. Geri N, Geri Y. The Information Age Measurement Paradox: Collecting Too Much Data. Informing Science 2011; 14: 47-59.

7. Ahmed AH, Giri J, Kashyap R, Singh B, Dong Y, Kilickaya O, Erwin PJ, Murad MH, Pickering BW. Outcome of Adverse Events and Medical Errors in the Intensive Care Unit: A Systematic Review and Metaanalysis. American journal of medical quality: the official journal of the American College of Medical Quality 2013; Dec 19. PubMed PMID: 24357344.

8. Wusthoff CJ, Shellhaas RA, Clancy RR. Limitations of single-channel EEG on the forehead for neonatal seizure detection. J Perinatol 2009; 29(3): 237-242. PubMed PMID: 19052554. Epub 2008/12/05.

9. Akman CI, Micic V, Thompson A, Riviello JJ, Jr. Seizure detection using digital trend analysis: Factors affecting utility. Epilepsy Res 2011; 93(1): 66-72. PubMed PMID: 21146370. Epub 2010/12/15.

10. Sackellares JC, Shiau DS, Halford JJ, LaRoche SM, Kelly KM. Quantitative EEG analysis for automated detection of nonconvulsive seizures in intensive care units. Epilepsy Behav 2011; 22 (Suppl. 1): S69-S73. PubMed PMID: 22078521. Epub 2011/12/07.

11. Akman CI, Riviello JJ, Jr. Generalized periodic epileptiform discharges in critically ill children: a continuum of status epilepticus or an epiphenomenon? J Clin Neurophysiol 2011; 28(4): 366-372. PubMed PMID: 21811125. Epub 2011/08/04.

12. Snodgrass SM, Tsuburaya K, Ajmone-Marsan C. Clinical significance of periodic lateralized epileptiform discharges: relationship with status epilepticus. J Clin Neurophysiol 1989; 6(2): 159-172. PubMed PMID: 2708516. Epub 1989/04/01.

13. Health USDo, Human Services USA. Protecting the privacy of patients' health information. Summary of the final regulation. Health care law monthly 2000: 20-24. PubMed PMID: 11924156.

14. Tuckett AG. Applying thematic analysis theory to practice: a researcher's experience. Contemporary nurse 2005; 19(1-2): 75-87. PubMed PMID: 16167437.

15. Gershon E. Gulrit. Haifa, Israel: Technion Institute of Technology; 2013.

16.van Putten MJ. The colorful brain: visualization of EEG background patterns. Journal of clinical neurophysiology : official publication of the American Electroencephalographic Society 2008; 25(2): 63-68. PubMed PMID: 18340270.

17.Spooner SA. Special requirements of electronic health record systems in pediatrics. Pediatrics 2007; 119(3): 631-637. PubMed PMID: 17332220. Epub 2007/03/03.

18. Hemphill JC, Andrews P, De Georgia M. Multimodal monitoring and neurocritical care bioinformatics. Nature reviews Neurology 2011; 7(8): 451-460. PubMed PMID: 21750522.

19.Zeng Q, Cimino JJ. A knowledge-based, concept-oriented view generation system for clinical data. Journal of biomedical informatics 2001; 34(2): 112-128. PubMed PMID: 11515411.

20.Pickering BW, Gajic O, Ahmed A, Herasevich V, Keegan MT. Data utilization for medical decision making at the time of patient admission to ICU. Critical care medicine 2013; 41(6): 1502-1510. PubMed PMID: 23528804 .

21. Siebig S, Kuhls S, Imhoff M, Gather U, Scholmerich J, Wrede CE. Intensive care unit alarms -how many do we need? Critical care medicine 2010; 38(2): 451-456. PubMed PMID: 20016379.

22. Mitka M. Joint Commission Warns of Alarm Fatigue Multitude of Alarms From Monitoring Devices Problematic. Jama-J Am Med Assoc 2013; 309(22): 2315-2316. PubMed PMID: WOS:000320176000003.

23. Akre M, Finkelstein M, Erickson M, Liu M, Vanderbilt L, Billman G. Sensitivity of the pediatric early warning score to identify patient deterioration. Pediatrics 2010; 125(4): e763-e769. PubMed PMID: 20308222. 
24. Pollack MM, Patel KM, Ruttimann UE. PRISM III: an updated Pediatric Risk of Mortality score. Critical care medicine. 1996; 24(5): 743-752. PubMed PMID: 8706448.

25. Liu V, Turk BJ, Ragins AI, Kipnis P, Escobar GJ. An electronic Simplified Acute Physiology Score-based risk adjustment score for critical illness in an integrated healthcare system. Critical care medicine 2013; 41(1): 41-48. PubMed PMID: 23222263.

26. Wilkinson L, Wills G. The grammar of graphics. 2nd ed. New York: Springer; 2005. xviii, 690 p. p.

27. Starren J, Johnson SB. An object-oriented taxonomy of medical data presentations. J Am Med Inform Assoc 2000; 7(1): 1-20. PubMed PMID: 10641959. Pubmed Central PMCID: 61451.

28. Sebastian K, Sari V, Loy LY, Zhang F, Zhang Z, Feng M. Multi-signal Visualization of Physiology (MVP): a novel visualization dashboard for physiological monitoring of Traumatic Brain Injury patients. Conference proceedings: Annual International Conference of the IEEE Engineering in Medicine and Biology Society IEEE Engineering in Medicine and Biology Society Conference 2012; 2012: 2000-3. PubMed PMID: 23366310.

29. Bennett KB, Flach JM. Graphical Displays - Implications for Divided Attention, Focused Attention, and Problem-Solving. Hum Factors 1992; 34(5): 513-533. PubMed PMID: WOS:A1992KA54500002.

30. Wickens CD, Merwin DH, Lin EL. Implications of Graphics Enhancements for the Visualization of Scientific-Data - Dimensional Integrality, Stereopsis, Motion, and Mesh. Hum Factors 1994; 36(1): 44-61. PubMed PMID: WOS:A1994NN52200003.

31. Abend NS, Chapman KE, Gallentine WB, Goldstein J, Hyslop AE, Loddenkemper T, Nash KB, Riviello JJ, Jr., Hahn CD, Pediatric Critical Care EEGG, the Critical Care EEGMRC. Electroencephalographic monitoring in the pediatric intensive care unit. Current neurology and neuroscience reports 2013; 13(3): 330. PubMed PMID: 23335026. Pubmed Central PMCID: 3569710.

32. Hansen JP. An experimental investigation of configural, digital, and temporal information on process displays. Hum Factors 1995; 37(3): 539-552. PubMed PMID: WOS:A1995TN43600006.

33. Carswell CM, Wickens CD. Mixing and matching lower-level codes for object displays: Evidence for two sources of proximity compatibility. Hum Factors 1996; 38(1): 1-22. PubMed PMID: WOS:A1996UP37500001.

34.Drews FA, Westenskow DR. The right picture is worth a thousand numbers: Data displays in anesthesia. Hum Factors 2006 Spr; 48(1): 59-71. PubMed PMID: WOS:000237042500007. 